\title{
El tabaco y su influencia en el periodonto
}

\author{
LORDELO MJ*
}

Lordelo MJ. El tabaco y su influencia en el periodonto. Av Periodon Implantol. 2005; 17, 1:221-228.

\begin{abstract}
RESUMEN
La periodontitis es una enfermedad multifactorial. El tabaquismo es uno de los factores ambientales que cambia más la susceptibilidad del individuo al desarrollo de la enfermedad periodontal.
\end{abstract}

Análogamente a lo que ocurre a nivel de la salud en general, el tabaco provoca efectos adversos graves en la cavidad bucal, en particular en los tejidos periodontales.

Los datos epidemiológicos cogidos de la literatura fornecen información importante que está en concordancia con la mayor parte de los criterios de Bradford Hill para el establecimiento de una relación causal entre el tabaco y la periodontitis.

La patogénesis de la destrucción periodontal es extremadamente compleja, ya que existen diversas vías clave relacionadas entre ellas que participan en el desencadenamiento y evolución de la enfermedad. El mecanismo de acción del tabaco puede ser explicado a través de la intervención en cada una de dichas vías.

Además de ser un factor de riesgo para la enfermedad, el tabaco perjudica la cicatrización y la respuesta al tratamiento básico y quirúrgico.

Será hecha una revisión bibliográfica de los trabajos publicados relacionados con el tema.

\section{PALABRAS CLAVE}

Tabaco; enfermedad periodontal; factor de riesgo; patogénesis; tratamiento periodontal; apoptósis

Aceptado para publicación: Febrero 2005

\section{INTRODUCCIÓN}

El tabaquismo es un hábito nocivo muy prevalente en la civilización. En todo el mundo, fuman en la actualidad alrededor de 1300 millones de personas. Esta cifra corresponde a $47,5 \%$ de los hombres y $10,3 \%$ de las mujeres. Como consecuencia la lista de enfermedades relacionadas con el tabaco tiende a aumentar y con ella la tasa de mortalidad. El tabaco es responsable de 1 de cada 10 muertes de personas adultas ( 5 millones de muertes cada año) y se cree que en el año de 2020 la cifra llegará a los 10 millones al año (1).

En Odontología, dos de los efectos adversos más graves son el cáncer oral y la enfermedad periodontal (2). La periodontitis es una enfermedad multifactorial y el tabaquismo constituye uno de los factores

* Práctica privada, Porto, Portugal 
ambientales que más influye en la susceptibilidad del individuo al desarrollo de la enfermedad (3).

El propósito de la presente revisión es averiguar: 1) que parámetros hacen del tabaco uno factor de riesgo para la enfermedad periodontal; 2) cual es el mecanismo de actuación a nivel de la patogénesis de la enfermedad; 3) de que modo perjudica la respuesta al tratamiento periodontal.

\section{MÉTODOS}

Se ha utilizado la MEDLINE/PubMed como motor de búsqueda para la pesquisa bibliográfica, utilizando las palabras clave "smoking" y "tobacco" en combinación con "periodontitis" y "periodontal disease". Para una búsqueda más específica y orientada para los temas en estudio se han introducido otras palabras clave como "risk factor", "microflora", "blood flow", "PMN", "interleukine", "citokine", "polymorphism", "periodontal therapy" y "apoptosis". También se ha hecho una búsqueda manual basada en las referencias bibliográficas de artículos de revisión recientes (entre 1999 y 2005). Además se han consultado algunos libros de texto para documentación adicional.

\section{EPIDEMIOLOGÍA}

Para que una variable sea considerada como un factor de riesgo, es necesario que se verifique una asociación causal entre la variable y la enfermedad. Los criterios de causalidad de Sir Bradford Hill (4) han sido utilizados para averiguar la relación entre el tabaco y la enfermedad periodontal.

Varios estudios epidemiológicos citados por Gelskey en 1999 (5) fornecen datos que están en concordancia con la mayor parte de estos criterios. Será echa una análisis de cada uno de los criterios de Hill basada en la metodología utilizada por esta autora y actualizada con los datos de publicaciones más recientes.

\section{Fuerza de Asociación}

La fuerza de asociación es mayor cuanto más elevado sea el valor del odds ratio (OR) o riesgo relativo (RR). Se ha observado en numerosos estudios una asociación positiva, pero con una magnitud de fuerza bastante variable - OR desde 1,4 hasta 25,64 (3;6-16). La discrepancia puede ser explicada por la variación del diseño del estudio, de la definición de enfermedad, de los valores umbrales, de los métodos de medición, de la populación estudiada y del controlo de factores como la edad, el sexo y la higiene.
Una meta-análisis de seis estudios ha evidenciado que el hecho de fumar aumenta casi tres veces la probabilidad de desarrollar periodontitis avanzada (17).

El Nacional Health and Nutrition Examination Survey (NHANES) III ha aportado datos para el estudio epidemiológico más largo y reciente sobre tabaquismo y periodontitis. Los resultados son bastante fidedignos considerando la representatividad de la muestra $(12,329$ estadounidenses adultos). Se ha observado que el tabaco era responsable de la periodontitis en la mitad de los casos y que los fumadores eran cuatro veces más propensos a tener la enfermedad (6).

\section{Consistencia}

A pesar de las diferentes condiciones de los estudios existe un resultado consistente en la literatura ya que en todos ellos se demuestra una asociación positiva entre el factor potencial, factor de riesgo y la enfermedad. Los estudios transversales (3;7-10), los casos y controles (11), los cohortes (12) y más recientemente los longitudinales (13-15) han relacionado el tabaquismo con una mayor prevalencia de los señales clínicos de enfermedad (pérdida de inserción, profundidad al sondaje y pérdida ósea).

\section{Especificidad}

El tabaco es considerado un factor específico cuando, tras la eliminación del hábito se produce un efecto periodontal favorable. En un estudio longitudinal se observa que la pérdida ósea es más lenta con la cesación del hábito nocivo (13). Un comportamiento de los tejidos comparable entre no-fumadores y ex-fumadores sugiere que la eliminación del tabaco favorece la salud periodontal (18).

\section{Temporalidad}

La relación de temporalidad ha sido evidenciada en estudios longitudinales donde, en la secuencia de exposición al agente causal existe una mayor progresión de la enfermedad (13-15) y una respuesta al tratamiento inadecuada (18;19). Por otro lado, el "precio" por el echo de ser fumador ha sido calculado en 27 años de progresión de la enfermedad relativamente a la pérdida de inserción periodontal (9).

\section{Gradiente Biológico}

Se ha constatado que cuanto mayor es el número de cigarrillos consumidos al día o de paquetes al año, mayor es el riesgo de desarrollar la enfermedad $(7 ; 8)$. 
Así se ha establecido una relación directa entre la dosis y el efecto.

\section{Plausibilidad Biológica}

Existe actualmente una base teórica que ofrece plausibilidad a la asociación entre el tabaco y la periodontitis. En otras palabras, el conocimiento actual de un proceso biopatogénico por lo cual el tabaco interviene en el periodonto puede dar una explicación biológica a la asociación. Este proceso será analizado con detalle más adelante en el artículo.

\section{Analogía}

Se ha comprobado que el tabaquismo esta directamente implicado en otras enfermedades. Por lo menos $30 \%$ de las muertes por cáncer son provocadas por el tabaco. Este hábito nocivo es la causa principal de cáncer del pulmón, de la laringe, de la cavidad bucal, de la faringe y del esófago, Además el tabaco es causa de otras enfermedades igualmente graves como la enfermedad cardiovascular, bronquitis enfisema y enfarte (20).

\section{Experimentación}

Para comprobar que el tabaco es una causa de la periodontitis seria necesario hacer estudios controlados aleatorizados prospectivos y estudios con un diseño experimental riguroso. Si embargo estos estudios implicarían someter los individuos a la exposición de un factor de riesgo, y eso sería éticamente inaceptable.

\section{INTERVENCIÓN DEL TABACO A NIVEL DE LA MICROFLORA}

Con relación a la patogenicidad de la microflora, existen diferentes estudios con resultados contradictorios (21-27). Sin embargo hay publicaciones donde se verifica una correlación positiva entre las dos variables.

Se ha demuestrado que los fumadores presentan una mayor probabilidad de infección con bacterias patogénicas (Porphyromona gingivals, Tanerella forsythensis, Prevotella intermedia, Peptostreptococcus micros, Fusobacterium nucleatum, Campylobacter rectus) $(23 ; 25)$ y mayor prevalencia de flora exógena (Escherichia coli, Candida albicans) (24). Estos resultados pueden ser consecuencia de una disminución de la presión de oxígeno en la bolsa (efecto local del humo del tabaco), que favorece crecimiento de anae- robios (28). Existe también una teoría que defiende que las bacterias P.g., A.a., T.f. son más difíciles de remover en fumadores $(18 ; 29)$.

\section{INTERVENCIÓN DEL TABACO A NIVEL DE LA RESPUESTA DEL HUÉSPED}

\section{Vascularización}

Se ha observado que los fumadores presentan una respuesta inflamatoria retardada o disminuida. Hay menor sangrado al sondage y menor rubor de la encía $(30 ; 31)$. Las bolsas de los sujetos fumadores presentan una menor cantidad de fluido crevicular gingival (32). La explicación para estos factos no está clarificada todavía. Esto parece ser debido a efectos a largo plazo de la nicotina en la inflamación y no a su efecto vasoconstrictor local, como se había creído antes $(33 ; 34)$. La hipótesis de que la disminución de la tendencia al sangrado puede estar relacionada con la menor densidad vascular o con una menor cantidad de vasos ha sido testada pero con resultados contradictorios $(35 ; 36)$.

\section{Sistema Inmunitario}

Uno de los mecanismos por los cuales el tabaco puede intervenir en la patogénesis de la enfermedad periodontal es cambiando la capacidad de defensa del organismo.

Con relación a la primera línea de defensa, los estudios son bastante conclusivos. En 1977, Kenney y sus colaboradores han observado que los neutrofilos de los fumadores presentaban una menor capacidad de fagocitosis y una menor viabilidad (37). En la realidad el tabaco perjudica no solo la fagocitosis pero también otras funciones de los polimorfonucleares, como la quimiotaxis, la producción del inhibidor de la proteasa, la generación de superoxido y peroxido de hidrógeno, y la expresión de moléculas de adhesión, tiendo como consecuencia una actividad defensiva deficiente y una mayor destrucción tisular (38-41).

En lo que respecta a la segunda linea de defensa todavía hay mucho por investigar. Se sabe que los linfocitos de los fumadores presentan una menor capacidad proliferativa y una menor producción de anticuerpos $(42 ; 43)$. Directamente relacionado con la periodontitis, el estudio de Tangada ha evidenciado una disminución de IgG2 específica para el Actinobacillus actinomycetemcomitans (44).

Se ha demuestrado que el tabaco provoca un desequilibrio en la producción y actuación de la comple- 
ja red de citoquinas pro-inflamatorias. Sin embargo, los estudios no son todavía conclusivos, ya que los datos disponibles son bastante contradictorios. Se ha observado que la nicotina sola y en la presencia de Lipopolissacárido estimula la secreción de PGE2, $(45 ; 46)$ de IL-6 y de IL-8 (47). Algunos autores han llegado a una asociación positiva entre el tabaco y el TNF- $\alpha,(48)$. Más recientemente esta asociación se observa también con las IL-1 $\beta$ y IL-6 (49). No obstante, existen publicaciones con resultados distintos en lo respecta a la IL-1 $\beta(50 ; 51)$, a la IL-6 y al TNF$\alpha .(52)$.

En resumen, el papel del tabaco en la patogénesis de la enfermedad periodontal tiene todavía diversos puntos por aclarar. Sin embargo, por la que se ha buscado en la literatura, se puede concluir que el tabaco potencia la enfermedad periodontal a través de su intervención en lo que se consideran los puntos clave del paradigma de la patogénesis de la enfermedad periodontal (Fig. 1).

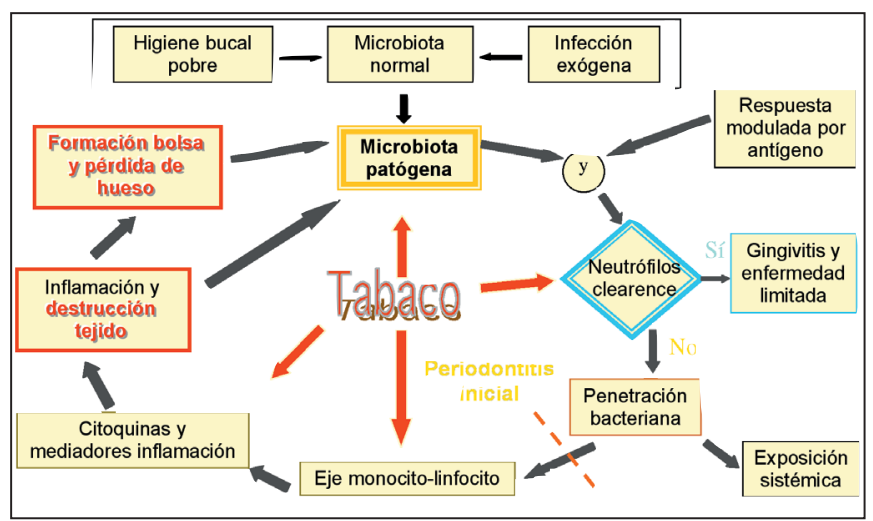

Fig. 1 - Patogénesis de la destrucción periodontal en relación con el tabaco (adaptación del diagrama presentado por Offenbacher en 1996 (53)).

\section{Interacción gen-ambiente}

El polimorfismo genético puede, interactuando con los factores de riesgo ambientales, alterar la susceptibilidad del individuo por el desarrollo de la periodontitis. Uno de los genes estudiado está implicado en el metabolismo de los productos del humo del tabaco. Es el NAT2, que codifica la enzima N-acetiltrasferasa. La traducción fenotípica consiste en la variación de la velocidad del metabolismo. El NAT2 positivo ("lento") ha sido asociado a un mayor riesgo de enfermedad y a severidad de pérdida ósea, principalmente en fumadores (54). Otro de los candidatos a factor de susceptibilidad genética asociado al tabaco es el IL-1. Se ha observado que el genotipo positivo aumenta el riesgo de periodontitis en fumadores, mientras que en no fumadores este aumento no ocurre $(55 ; 56)$.

\section{INTERVENCIÓN DEL TABACO A NIVEL DE LA CICATRIZACIÓN Y RESPUESTA AL TRATAMIENTO}

Los resultados clínicos ponen en evidencia la respuesta al tratamiento inferior. MacFarlane ha llegado a cifras bastante significativas - $90 \%$ de las periodontitis refractarias fueron encontradas en fumadores (38).

\section{Tratamiento no quirúrgico y quirúrgico}

Tras el raspado y alisado radicular se ha observado que los pacientes fumadores presentaban una menor reducción de la profundidad de bolsa con una diferencia estadísticamente significativa (18), (57-59). No obstante, con relación al nivel de inserción las diferencias entre fumadores y no fumadores no son significativas. El echo de que los fumadores no presenten señales inflamatorios tan evidentes (31) puede resultar en una menor disminución del edema tras el tratamiento y explicar estos resultados.

$\mathrm{Al}$ estudiar la respuesta al tratamiento con colgajo de acceso, y con regeneración tisular guiada, se ha observado una gran incapacidad de cicatrización y de regeneración de los tejidos en fumadores menor reducción de la profundidad de la bolsa y menor ganancia de inserción $(19 ; 60 ; 61)$. La respuesta al tratamiento inadecuada puede ser explicada por la intervención del tabaco a nivel de la microflora y respuesta del huésped, como se ha analizado antes. Se ha observado que, tras la cirugía periodontal, el tabaco provoca un desequilibrio entre las proteasas y sus inhibidores, perjudicando la cicatrización (62). Además el tabaco interviene en la capacidad de adhesión de los fibroblastos del ligamiento periodontal a la superficie radicular $(63 ; 64)$.

Con el objetivo de mejorar la respuesta al tratamiento en fumadores se han hecho ensayos clínicos utilizando agentes químicos (triclosan y doxiciclina) como coadyuvantes de la terapia no quirúrgica con resultados satisfactorios $(19 ; 65 ; 66)$.

\section{DISCUSIÓN Y CONCLUSIÓN}

Con base en una análisis epidemiológica, Hujoel (67) sugiere que el echo de reconocer tardíamente el efecto del tabaco en la enfermedad periodontal hizo que, en el siglo XX, una periodontitis epidémica haga permanecido velada. Así, el entendimiento de la etiología y tratamiento de la periodontitis habrá sido destorcido. De hecho se ha comprobado la importancia del tabaco no solo como uno de los principales factores de riesgo para el desencadenamiento y progresión 
de la enfermedad periodontal pero también como un impedimento a una respuesta normal al tratamiento periodontal.

Hay todavía mucho por aclarar en este tema, principalmente con relación al mecanismo por lo cual el tabaco interviene en la progresión de la enfermedad y respuesta al tratamiento.

Recientemente se ha investigado el papel de la apoptósis en la enfermedad periodontal (68). Análogamente a la inducción de la apoptósis por el tabaco en otros tejidos (69-73), puede que la muerte celular programada sea inducida por este agente también en el periodonto y intervenga, por lo tanto en otra vía de la patogénesis de la enfermedad periodontal.

\section{ABSTRACT}

Periodontitis is a multifactorial disease. Smoking is one of the environmental factors that most changes the susceptibility of the individual to the development of the periodontal disease.

Analogous to what happens at the level of general health, tobacco causes serious adverse effects in the oral cavity, namely in periodontal tissues.

The epidemiologic data taken from literature gives important information that is in agreement with the greater part of the criteria of Bradford Hill for the establishment of a causal relationship between tobacco and periodontitis.

The pathogenesis of the periodontal destruction is extremely complex, since there are diverse critical pathways interconnected between them which participate in the outbreak and evolution of the disease. The mechanism of action of tobacco can be explained by its intervention in each of these pathways.

In addition to being a risk factor for the disease, tobacco impairs healing and the response to basic and surgical treatment.

Bibliographical revision of the published works related to the subject will be carried out.

\section{KEY WORDS}

Smoking; periodontal disease; risk factor; pathogenesis; periodontal therapy; apoptosis

\section{CORRESPONDENCIA}

Maria João Lordelo

R. João Rosa, 152, A 63

4460-189 SRA. DA HORA

PORTUGAL

mjlordelo@yahoo.com

\section{BIBLIOGRAFíA}

1.WHO Smoking Free Initiative http://www.who.int/ tobacco/en/.

2. Johnson NW, Bain CA. Tobacco and oral disease. EUWorking Group on Tobacco and Oral Health. Br Dent J 2000 August 26; 189 (4): 200-6.

3. Haber J, Wattles J, Crowley M, Mandell R, Joshipura K, Kent RL. Evidence for cigarette smoking as a major risk factor for periodontitis. J Periodontol 1993 January; 64 (1): 16-23.

4. QVHS Dictionary and Reference: Hill's Criteria http://www.qvhs.com.au/Dict-Ref/hillcrit.htm.

5. Gelskey SC. Cigarette smoking and periodontitis: methodology to assess the strength of evidence in support of a causal association. Community Dent Oral Epide-miol 1999 February; 27 (1): 16-24.

6. Tomar SL, Asma S. Smoking-attributable periodontitis in the United States: findings from NHANES III. National Health and Nutrition Examination Survey. J Periodontol 2000 May; 71 (5): 743-51.

7. Grossi SG, Zambon JJ, Ho AW, Koch G, Dunford RG, Machtei EE, Norderyd OM, Genco RJ. Assessment of risk for periodontal disease. I. Risk indicators for attachment loss. J Periodontol 1994 March;65(3):260-7.

8. Martinez-Canut P, Lorca A, Magan R. Smoking and periodontal disease severity. J Clin Periodontol 1995 October; 22 (10): 743-9.

9. Haffajee AD, Socransky SS. Relationship of cigarette smoking to attachment level profiles. J Clin Periodontol 2001 April; 28 (4): 283-95.

10. Amarasena N, Ekanayaka AN, Herath L, Miyazaki H. Tobacco use and oral hygiene as risk indicators for periodontitis. Community Dent Oral Epidemiol 2002 April; 30 (2): 115-23.

11. Calsina G, Ramon JM, Echeverria JJ. Effects of smoking on periodontal tissues. J Clin Periodontol 2002 August; 29 (8): 771-6. 
12. Bergstrom J. Tobacco smoking and risk for periodontal disease. J Clin Periodontol 2003 February; 30 (2): 107-13.

13. Bolin A, Eklund G, Frithiof L, Lavstedt S. The effect of changed smoking habits on marginal alveolar bone loss. A longitudinal study. Swed Dent J 1993; 17 (5): 21 1-6.

14. Bergstrom J, Eliasson S, Dock J. A 10-year prospective study of tobacco smoking and periodontal health. J Periodontol 2000 August;71 (8): 1338-47.

15. Bergstrom J. Influence of tobacco smoking on periodontal bone height. Long-term observations and a hypothesis. J Clin Periodontol 2004 April; 31 (4): 260-6.

16. Hyman JJ, Reid BC. Epidemiologic risk factors for periodontal attachment loss among adults in the United States. J Clin Periodontol 2003 March;30 (3): 230-7.

17. Papapanou PN. Periodontal diseases: epidemiology. Ann Periodontol 1996 November; 1 (1): 1-36.

18. Grossi SG, Zambon J, Machtei EE, Schifferle R, Andreana S, Genco RJ, Cummins D, Harrap G. Effects of smoking and smoking cessation on healing after mechanical periodontal therapy. J Am Dent Assoc 1997 May; 128 (5): 599-607.

19. Scabbia A, Cho KS, Sigurdsson TJ, Kim CK, Trombelli L. Cigarette smoking negatively affects healing response following flap debridement surgery. J Periodontol 2001 January; 72 (1): 43-9.

20. ACS: Cigarette Smoking http://www.cancer.org/docroot/PED/content/PED_10_2X_Cigarette_Smoking_and_C ancer.asp?sitearea $=\mathrm{PED}$.

21. Stoltenberg JL, Osborn JB, Pihlstrom BL, Herzberg MC, Aeppli DM, Wolff LF, Fischer GE. Association between cigarette smoking, bacterial pathogens, and periodontal status. J Periodontol 1993 December; 64 (12): 1225-30.

22. Preber H, Bergstrom J, Linder LE. Occurrence of periopathogens in smoker and non-smoker patients. J Clin Periodontol 1992 October; 19 (9 Pt 1): 667-71.

23. Zambon JJ, Grossi SG, Machtei EE, Ho AW, Dunford R, Genco RJ. Cigarette smoking increases the risk for subgingival infection with periodontal pathogens. J Periodontol 1996 October; 67 (10 Suppl): 1050-4.

24. Kamma JJ, Nakou M, Baehni PC. Clinical and microbiological characteristics of smokers with early onset periodontitis. J Periodontal Res 1999 January; 34 (1): 25-33.

25. van Winkelhoff AJ, Bosch-Tijhof CJ, Winkel EG, van der Reijden WA. Smoking affects the subgingival microflora in periodontitis. J Periodontol 2001 May; 72 (5): 666-71.
26. Haffajee AD, Socransky SS. Relationship of cigarette smoking to the subgingival microbiota. J Clin Perio-dontol 2001 May; 28 (5): 377-88.

27. Mager DL, Haffajee AD, Socransky SS. Effects of periodontitis and smoking on the microbiota of oral mucous membranes and saliva in systemically healthy subjects. J Clin Periodontol 2003 December; 30 (12): 1031-7.

28. Hanioka T, Tanaka M, Takaya K, Matsumori Y, Shizukuishi S. Pocket oxygen tension in smokers and non-smokers with periodontal disease. J Periodontol 2000 April; 71 (4): 550-4.

29. Renvert S, Dahlen G, Wikstrom M. The clinical and microbiological effects of non-surgical periodontal therapy in smokers and non-smokers. J Clin Periodontol 1998 February; 25 (2): 153-7.

30. Preber H, Bergstrom J. Occurrence of gingival bleeding in smoker and non-smoker patients. Acta Odontol Scand 1985 October; 43 (5): 315-20.

31. Bergstrom J, Bostrom L. Tobacco smoking and periodontal hemorrhagic responsiveness. J Clin Periodontol 2001 July; 28 (7): 680-5.

32. Bergstrom J, Preber $\mathrm{H}$. The influence of cigarette smoking on the development of experimental gingivitis. J Periodontal Res 1986 November; 21 (6): 668-76.

33. Meekin TN, Wilson RF, Scott DA, Ide M, Palmer RM. Laser Doppler flowmeter measurement of relative gingival and forehead skin blood flow in light and heavy smokers during and after smoking. J Clin Periodontol 2000 April; 27 (4): 236-42.

34. Mavropoulos A, Aars H, Brodin P. Hyperaemic response to cigarette smoking in healthy gingiva. J Clin Periodontol 2003 March; 30 (3): 214-21.

35. Rezavandi K, Palmer RM, Odell EW, Scott DA, Wilson RF. Expression of ICAM-1 and E-selectin in gingival tissues of smokers and non-smokers with periodontitis. J Oral Pathol Med 2002 January; 31 (1): 59-64.

36. Sonmez S, Canda T, Ozkara E, Ak D. Quantitative evaluation of the vasculature and fibronectin localization in gingival connective tissue of smokers and non-smokers. J Periodontol 2003 June; 74 (6): 822-30.

37. Kenney EB, Kraal JH, Saxe SR, Jones J. The effect of cigarette smoke on human oral polymorphonuclear leukocytes. J Periodontal Res 1977 July; 12 (4): 227-34.

38. MacFarlane GD, Herzberg MC, Wolff LF, Hardie NA. Refractory periodontitis associated with abnormal polymorphonuclear leukocyte phagocytosis and cigarette 
smoking. J Periodontol 1992 November; 63 (11): 908-13.

39. Pabst MJ, Pabst KM, Collier JA, Coleman TC, LemonsPrince ML, Godat MS, Waring MB, Babu JP. Inhibition of neutrophil and monocyte defensive functions by nicotine. J Periodontol 1995 December; 66 (12): 1047-55.

40. Ryder MI, Fujitaki R, Johnson G, Hyun W. Alterations of neutrophil oxidative burst by in vitro smoke exposure: implications for oral and systemic diseases. Ann Periodontol 1998 July; 3 (1): 76-87.

41. Palmer RM, Scott DA, Meekin TN, Poston RN, Odell EW, Wilson RF. Potential mechanisms of susceptibility to periodontitis in tobacco smokers. J Periodontal Res 1999 October; 34 (7): 363-9.

42. Quinn SM, Zhang JB, Gunsolley JC, Schenkein JG, Schenkein HA, Tew JG. Influence of smoking and race on immunoglobulin $\mathrm{G}$ subclass concentrations in earlyonset periodontitis patients. Infect Immun 1996 July; 64 (7): 2500-5.

43. Quinn SM, Zhang JB, Gunsolley JC, Schenkein HA, Tew JG. The influence of smoking and race on adult periodontitis and serum IgG2 levels. J Periodontol 1998 February; 69 (2): 171-7.

44. Tangada SD, Califano JV, Nakashima K, Quinn SM, Zhang JB, Gunsolley JC, Schenkein HA, Tew JG. The effect of smoking on serum IgG2 reactive with Actinobacillus actinomycetemcomitans in early-onset periodontitis patients. J Periodontol 1997 September; 68 (9): 842-50.

45. Payne JB, Johnson GK, Reinhardt RA, Dyer JK, Maze CA, Dunning DG. Nicotine effects on PGE2 and IL-1 beta release by LPS-treated human monocytes. J Periodontal Res 1996 February; 31 (2): 99-104.

46. Bernzweig E, Payne JB, Reinhardt RA, Dyer JK, Patil KD. Nicotine and smokeless tobacco effects on gingival and peripheral blood mononuclear cells. J Clin Periodontol 1998 March; 25 (3): 246-52.

47. Wendell KJ, Stein SH. Regulation of cytokine production in human gingival fibroblasts following treatment with nicotine and lipopolysaccharide. J Periodontol 2001 August; 72 (8): 1038-44.

48. Bostrom L, Linder LE, Bergstrom J. Smoking and cervicular fluid levels of IL-6 and TNF-alpha in periodontal disease. J Clin Periodontol 1999 June; 26 (6): 352-7.

49. Kamma JJ, Giannopoulou C, Vasdekis VG, Mombelli A. Cytokine profile in gingival crevicular fluid of aggressive periodontitis: influence of smoking and stress. J Clin Periodontol 2004 October; 31 (10): 894-902.
50. Bostrom L, Linder LE, Bergstrom J. Smoking and GCF levels of IL-1 beta and IL-lra in periodontal disease. J Clin Periodontol 2000 April; 27 (4): 250-5.

51. Rawlinson A, Grummitt JM, Walsh TF, Ian Douglas CW. Interleukin 1 and receptor antagonist levels in gingival crevicular fluid in heavy smokers versus non-smokers. J Clin Periodontol 2003 January; 30 (1): 42-8.

52. Erdemir EO, Duran I, Haliloglu S. Effects of smoking on clinical parameters and the gingival crevicular fluid levels of IL-6 and TNF-alpha in patients with chronic periodontitis. J Clin Periodontol 2004 February; 31 (2): 99-104.

53. Offenbacher S. Periodontal diseases: pathogenesis. Ann Periodontol 1996 November;1(1):821-78.

54. Kocher T, Sawaf H, Fanghanel J, Timm R, Meisel P. Association between bone loss in periodontal disease and polymorphism of $\mathrm{N}$-acetyltransferase (NAT2). J Clin Periodontol 2002 January; 29 (1): 21-7.

55. Meisel P, Siegemund A, Dombrowa S, Sawaf H, Fanghaenel J, Kocher T. Smoking and polymorphisms of the interleukin-l gene cluster (IL-lalpha, IL-1beta, and IL-lRN) in patients with periodontal disease. J Periodontol 2002 January; 73 (1): 27-32.

56. Meisel P, Schwahn C, Gesch D, Bernhardt O, John U, Kocher T. Dose-effect relation of smoking and the interleukin-l gene polymorphism in periodontal disease. J Periodontol 2004 February; 75 (2): 236-42.

57. Palmer RM, Matthews JP, Wilson RF. Non-surgical periodontal treatment with and without adjunctive metronidazole in smokers and non-smokers. J Clin Periodontol 1999 March; 26 (3): 158-63.

58. Darby IB, Hodge PJ, Riggio MP, Kinane DF. Clinical and microbiological effect of scaling and root planing in smoker and non-smoker chronic and aggressive periodontitis patients. J Clin Periodontol 2005 February; 32 (2): 200-6.

59. Labriola A, Needleman I, Moles DR. Systematic review of the effect of smoking on nonsurgical periodontal therapy. Periodontol 2000 2005; 37: 124-37.

60. Trombelli L, Cho KS, Kim CK, Scapoli C, Scabbia A. Impaired healing response of periodontal furcation defects following flap debridement surgery in smokers. A controlled clinical trial. J Clin Periodontol 2003 January; 30 (1): 81-7.

61. Stavropoulos A, Mardas N, Herrero F, Karring T. Smoking affects the outcome of guided tissue regeneration with bioresorbable membranes: a retrospective analysis of 
intrabony defects. J Clin Periodontol 2004 November; 31 (11): 945-50.

62. Persson L, Bergstrom J, Gustafsson A. Effect of tobacco smoking on neutrophil activity following periodontal surgery. J Periodontol 2003 October; 74 (10): 1475-82.

63. James JA, Sayers NM, Drucker DB, Hull PS. Effects of tobacco products on the attachment and growth of periodontal ligament fibroblasts. J Periodontol 1999 May; 70 (5): 518-25.

64. Gamal AY, Bayomy MM. Effect of cigarette smoking on human PDL fibroblasts attachment to periodontally involved root surfaces in vitro. J Clin Periodontol 2002 August; 29 (8): 763-70.

65. Kerdvongbundit V, Wikesjo UM. Effect of triclosan on healing following non-surgical periodontal therapy in smokers. J Clin Periodontol 2003 December; 30 (12): 1024-30.

66. Tomasi C, Wennstrom JL. Locally delivered doxycycline improves the healing following non-surgical periodontal therapy in smokers. J Clin Periodontol 2004 August; 31 (8): 589-95.

67. Hujoel PP, del Aguila MA, DeRouen TA, Bergstrom J. A hidden periodontitis epidemic during the 20th century? Community Dent Oral Epidemiol 2003 February; 31 (1): 1-6.
68. Bascones A, Gamonal J, Gomez M, Silva A, Gonzalez MA. New knowledge of the pathogenesis of periodontal disease. Quintessence Int 2004 October; 35 (9): 706-16.

69. D’Agostini F, Balansky RM, Izzotti A, Lubet RA, Kelloff GJ, De FS. Modulation of apoptosis by cigarette smoke and cancer chemopreventive agents in the respiratory tract of rats. Carcinogenesis 2001 March; 22 (3): 375-80.

70. Hoshino Y, Mio T, Nagai S, Miki H, Ito I, Izumi T. Cytotoxic effects of cigarette smoke extract on an alveolar type II cell-derived cell line. Am J Physiol Lung Cell Mol Physiol 2001 August; 281 (2): L509-L516.

71. De FS, Balansky RM, D'Agostini F, Izzotti A, Camoirano A, Bennicelli C, Zhang Z, Wang Y, Lubet RA, You M. Molecular alterations and lung tumors in p53 mutant mice exposed to cigarette smoke. Cancer Res 2003 February 15; 63 (4): 793-800.

72. Schwartz JL, Muscat JE, Baker V, Larios E, Stephenson GD, Guo W, Xie T, Gu X, Chung FL. Oral cytology assessment by flow cytometry of DNA adducts, aneuploidy, proliferation and apoptosis shows differences between smokers and non-smokers. Oral Oncol 2003 December; 39 (8): 842-54.

73. DeMarini DM. Genotoxicity of tobacco smoke and tobacco smoke condensate: a review. Mutat Res 2004 November; 567 (2-3): 447-74. 